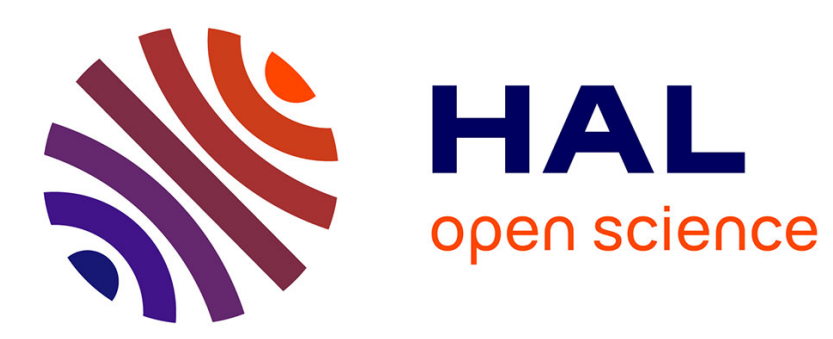

\title{
Une approche de la diversification discursive chez de jeunes enfants (30-48 mois)
}

\author{
Frédéric Torterat
}

\section{To cite this version:}

Frédéric Torterat. Une approche de la diversification discursive chez de jeunes enfants (30-48 mois).

Enfance, 2012, 2012 (02), pp.167 - 191. 10.4074/S0013754512002030 . hal-01706393

\section{HAL Id: hal-01706393 \\ https://hal.umontpellier.fr/hal-01706393}

Submitted on 11 Feb 2018

HAL is a multi-disciplinary open access archive for the deposit and dissemination of scientific research documents, whether they are published or not. The documents may come from teaching and research institutions in France or abroad, or from public or private research centers.
L'archive ouverte pluridisciplinaire HAL, est destinée au dépôt et à la diffusion de documents scientifiques de niveau recherche, publiés ou non, émanant des établissements d'enseignement et de recherche français ou étrangers, des laboratoires publics ou privés. 


\section{UNE APPROCHE DE LA DIVERSIFICATION DISCURSIVE CHEZ DE JEUNES ENFANTS (30-48 MOIS)

\author{
Frédéric Torterat
}

NecPlus | Enfance »

2012/2 N² 2 pages 167 à 191

ISSN 0013-7545

Article disponible en ligne à l'adresse :

https://www.cairn.info/revue-enfance2-2012-2-page-167.htm

\section{Pour citer cet article :}

Frédéric Torterat, « Une approche de la diversification discursive chez de jeunes enfants (30-48 mois) », Enfance 2012/2 ( $\left.\mathrm{N}^{\circ} 2\right)$, p. 167-191.

DOI 10.4074/S0013754512002030

Distribution électronique Cairn.info pour NecPlus.

(C) NecPlus. Tous droits réservés pour tous pays.

La reproduction ou représentation de cet article, notamment par photocopie, n'est autorisée que dans les limites des conditions générales d'utilisation du site ou, le cas échéant, des conditions générales de la licence souscrite par votre établissement. Toute autre reproduction ou représentation, en tout ou partie, sous quelque forme et de quelque manière que ce soit, est interdite sauf accord préalable et écrit de l'éditeur, en dehors des cas prévus par la législation en vigueur en France. Il est précisé que son stockage dans une base de données est également interdit. 


\title{
Une approche de la diversification discursive chez de jeunes enfants (30-48 mois)
}

\author{
Frédéric TORTERAT ${ }^{1}$
}

\section{RÉSUMÉ}

Cet article présente une approche des formes de diversification discursive chez de jeunes enfants (de 30 à 48 mois), en prenant appui sur des corpus oraux correspondant à des productions verbales recueillies en contexte d'apprentissage. Notre démarche a pour objet, d'une part, de montrer dans quelle mesure le traitement de ces données aboutit à déterminer les facteurs linguistiques les plus significatifs en acquisition, et, d'autre part, de mettre en regard les éléments qui contribuent aux variations interindividuelles apparaissant dans les productions discursives.

Afin d'illustrer l'éclairage qu'apporte ce type d'approche, nous la confronterons brièvement à une étude précédente au cours de laquelle nous avons pris en compte les capacités d'intervention spontanée des enfants en lien avec leur productivité lexicale. Cette étude, si elle s'est avérée significative en termes de profils d'apprentissage, n'a été qu'en partie satisfaisante en termes de profils d'acquisition. Un deuxième traitement des données, réparti sur cinq variables (apports thématiques, éléments rhématiques, verbes/amas verbaux, cadratifs et opérateurs), s'est révélé plus précis. Nous montrerons, en particulier, comment il permet d'analyser concrètement les manières dont les jeunes locuteurs diversifient effectivement leur expression.

MOTS CLEFS : DISCOURS, DIVERSIFICATION, ACQUISITION, APPRENTISSAGE, CORPUS, ORAL

${ }^{1}$ URE 03, Université de Nice Sophia Antipolis, 89 avenue George V, 06046 Nice Cédex 1. Email: frederic.torterat@unice.fr 


\section{ABSTRACT \\ Discursive Approach to variation in young children's oral productions (30 to 48 -month-old)}

This article presents a discursive approach of variation in young children's oral productions ( 30 to 48 -month-old). Based on dialogs, in pedagogic context, this work aims to show how analysis of these data contributes to select the more relevant domains in Discourse acquisition, consolidating simultaneously studies of interindividual variations.

To demonstrate the gain which represents this type of approach, we confront it briefly with a previous analysis, during which we took into account capacities of children's spontaneous interventions in connection with their lexical productivity. If this analysis seemed significant in terms of Learning Profiles, it was not satisfactory in terms of Acquisition Profiles. The second data processing, distributed between five variables (thematic supports, rhematic elements, verbs/verb clusters, cadratives and operators), showed itself more accurate as far as it allows to envisage more concretely the manners the young speakers diversify effectively their expression.

KEY-WORDS: DISCOURSE, DIVERSIFYING, ACQUISITION, LEARNING, CORPUS, ORAL DATA 


\section{Présentation de LA DÉmARCHe}

Apprendre à parler spontanément, c'est, pour l'enfant, recourir à de multiples opérations linguistiques, lesquelles interviennent non pas sur une ou quelquesunes, mais sur toutes les dimensions discursives qu'implique l'intervention d'un locuteur. Les productions concernées ne se réduisent pas à des alignements de mots plus ou moins intelligibles ou à ce qu'on appelle, ici et là, des " assemblages »: elles sont aussi la combinaison, en simultané, de groupes intonatifs, de domaines d'ordre (des mots), de constructions phrastiques, mais aussi de démarches discursives. Si les premiers champs d'acquisition ont été abondamment documentés par la recherche, comme nous le rappellerons en partie ci-après, celui de la diversification discursive, qui témoigne des manières dont l'enfant s'approprie divers types de conduites (inter)locutives et y recourt par lui-même, fait encore l'objet d'analyses très ponctuelles. Cette question apparaît pourtant au centre de l'apprentissage de la parole et des différents modes de socialisation qu'il induit.

Avant tout, rappelons que les recherches en linguistique de l'acquisition ont montré que, du côté des enfants, si la reconnaissance et la compréhension des mots hors contexte, laquelle s'accompagne généralement d'une détection des frontières prosodiques entre les groupes intonés, intervient vers 10-12 mois (Boysson-Bardies \& Vihman, 1991 ; Wauquier, 2005), les productions qui en témoignent n'apparaissent le plus couramment chez les sujets qu'à partir de 26-30 mois (Oller, 2000 ; Morgenstern \& Parisse, 2007). Ces productions coïncident généralement avec les premières combinaisons ordonnées de mots, ainsi qu'avec l'emploi et le réemploi, par les locuteurs, des marqueurs de structuration discursive (comme « et ", " après, moi (je) », " alors » ou « là » par exemple) : autant de manifestations concrètes qui révèlent, à leur juste mesure, que les jeunes enfants commencent à assimiler une diversité de plus en plus effective de conduites interlocutives. Dans ce domaine, une approche longitudinale de leurs productions orales permet d'aborder ce qui varie d'un locuteur à l'autre, mais également ce qui varie chez un même locuteur. Cela étant, elle est souvent confrontée à la multiplicité des facteurs à prendre en compte.

Bien que la question des variations intra-individuelles puisse elle aussi faire l'objet de plusieurs traitements en linguistique et en psycholinguistique (Saada-Robert \& Favrel, 2001 ; Bassano, 2005), il sera question ici d'un autre domaine d'étude qui concerne ce type d'acquisition (chez des enfants de L1), à savoir les variations interindividuelles. Vu le contexte de la recherche dont nous rendons compte ci-après, le fait que les productions abordées coïncident avec les premiers apprentissages scolaires envisageables dans plusieurs pays européens constitue une donnée importante, même si les travaux menés jusqu'ici plus particulièrement au sujet des marqueurs discursifs portent assez peu sur cette période (Mosegaard Hansen, 1998 ; Sanders \& Noordman, 2000).

Ce type d'analyse intégrant plusieurs variables, elle implique qu'on l'ait expérimentée antérieurement sur des corpus "de référence ", de manière à déterminer si les facteurs retenus sont significatifs ou non pour le traitement 
des données. C'est ce dont il sera question dans ces pages, où nous présentons une approche discursive inédite, que nous exemplifions, et dont nous décrivons, ensuite, les premiers résultats, les difficultés apparues au moment de l'analyse, ainsi que les perspectives de recherche qu'elle permet d'envisager.

\section{Exemple liminaire}

Pour illustrer ce qui justifie, de notre point de vue, la présente approche méthodologique, nous prendrons l'un des matériaux du corpus sur lequel nous nous appuyons, et sur lequel nous revenons ci-après. Il s'agit d'une réponse que formule un enfant de 46 mois, Maxim, en lien avec les quelques interventions qui précèdent, dans un dialogue avec d'autres locuteurs de son groupe (reportées infra):

bah les grandes fusées/ils vont décoller tous ensemble

Nous ne nous pencherons pas ici sur les éléments de prosodie, mais prise comme telle, cette production locutoriale correspond à une suite de mots dans laquelle interviennent des variables suprasegmentales, comme les intonations, les contours et les pauses, et dont la répartition est révélatrice de la manière dont le locuteur envisage des regroupements et des démarcations, mais aussi marque le contenu prédiqué d'une certaine expressivité (voir Martel, 2009). Si nous nous attardons en revanche sur le contenu lui-même, il apparaît que dans sa matérialité segmentale, linéaire, celui-ci témoigne de l'assimilation, par l'enfant, de certaines possibilités d'ordre des mots (ce qu'on appelle la topologie phrastique) : ainsi Maxim place-t-il le sujet pronominal « ils » devant la forme co-verbale « vont décoller », et fait-il précéder le pronom de sa désignation nominale « les grandes fusées » (dont il oublie ensuite, sans doute du fait de l'effort cognitif à produire, le paramètre du genre). De même rejette-t-il après l'infinitif le syntagme «tous ensemble », qui à la fois est apposé en partie au pronom «ils » et permet à l'enfant, de manière concomitante, de circonstancier le contenu exprimé par la forme coverbale. Cette combinaison, qu'ouvre une interjection onomatopéique («bah»), coïncide avec une production phrastique principalement basée sur une construction verbale intransitive dans laquelle le locuteur insiste notamment sur la position thématique du sujet. En résumé, l'enfant indique ce dont il parle (l'apport thématique), et fournit, à l'appui d'une phrase aboutie, certaines informations à son propos (les éléments rhématiques). Or, il ne le fait pas n’importe comment.

Effectivement, la description de ces caractéristiques n'est satisfaisante que si elle montre dans quelles proportions de telles variables se combinent. Une approche discursive complétera opportunément l'analyse qu'il est possible d'en effectuer, car l'enfant fait ici plus qu'intoner, ordonner des mots, construire une phrase verbale : il manifeste une démarche interlocutive à travers laquelle il montre ce qu'il a mémorisé des interventions dialogales qui précèdent, la manière dont il tente de se faire comprendre, et celle dont il s'efforce de s'imposer non pas seulement en tant que locuteur, mais aussi comme représentant d'un point de vue et comme participant d'un groupe social. Si l'on prend ce qui entoure cette production : 
Kyllian : bah qu'est-ce que je fais? / paw/paw paw

Ambre : c'est laquelle la plus grande?

Maxim : bah les grandes fusées/ils vont décoller tous ensemble

Ambre : c'est laquelle la plus grande?

Nous voyons que, d'une part chez Maxim, l'interjection («bah ») présente un caractère en partie réfutatif, tout en le confortant dans son rôle de participant : par cet intermédiaire, l'enfant intègre un moment discursif qui le dépasse mais dans lequel il tente de s'interposer. Par ailleurs, en « extraposant» l'élément thématique à l'avant de sa production (« les grandes fusées »), le jeune locuteur privilégie la désignation nominale au sujet de laquelle il fournit par la suite plusieurs indications. À ce titre, il donne une dimension focale à son propos.

Il y aurait d'autres caractéristiques à relever sur cette réponse que donne l'enfant. Rappelons seulement que ce caractère discursif de l'intervention du locuteur est central, surtout si l'on se place dans une perspective fonctionnaliste: non seulement une telle production témoigne de premières apparitions de formes, mais aussi de leur emploi de manière productive, autant d'éléments qu'il est possible d'envisager à travers un regard méthodologique qui facilite, à notre sens, l'évaluation des variations interindividuelles (Bassano, Maillochon, \& Eme 1998 ; Kail \& Bassano, 2000 ; Wauquier, 2003). Dans cette vue, l'analyse en termes d'emploi et de réemploi permet d'apporter des réponses concrètes à la question de l'impact de la diversification discursive sur l'appropriation de certaines conduites interlocutives représentées à l'oral. Cela apparaît d'autant plus clairement que les co-verbalisations y favorisent significativement les « reprises imitatives »(Veneziano, 2000) parmi les enfants, au cours notamment d'une organisation dialogale où ils ont plus ou moins leur part d'initiative.

\section{MÉTHODOLOGIE ET CORPUS}

\section{Participants et procédure}

La population d'enfants sur laquelle s'est appuyée la présente analyse correspond aux effectifs de trois classes multi-niveaux de maternelle, sur deux circonscriptions différentes de Nice (année 2007-2008, avec plusieurs milieux socio-économiques). Les classes en question, regroupant les petite et moyenne sections, ont rassemblé de 17 à 26 élèves âgés de 28-29 à 59 mois au moment du recueil des données. Au cours des séances qui ont été prises en compte, les élèves ont été répartis en groupes restreints de 5 à 8 sujets à l'occasion d'« ateliers tournants ", une organisation pédagogique qui permet de pratiquer plusieurs activités et d'accompagner plus favorablement les enfants. Sur les classes concernées, les activités consistaient soit dans un éveil sur les grandeurs et mesures, soit dans ce que les Programmes (2007, 2008) de l'école maternelle intitulent la " découverte du monde ", soit dans des essais en arts visuels. Nous avons enregistré les séances, le plus couramment de 6 à 25 minutes, pratiquées en présence des professeures des écoles responsables de la classe, les autres ateliers 
étant, pour l'un, pris en charge par l'ATSEM, et, pour l'autre, encadré par les deux intervenantes mais par intermittence ${ }^{1}$.

Nous partirons ici plus exactement du corpus de référence, lequel a été constitué d'après les enregistrements les plus satisfaisants. Pour ce corpus spécifique, lequel est représentatif de la démarche, les séances se sont étalées sur des périodes de 6 à 11 minutes et ont rassemblé des effectifs allant de 5 à 7 sujets, au cours d'ateliers de fabrication de petits objets individuels (la séance de « découverte du monde »). Lors de cette séance, les enfants ont été invités à construire des roulottes en utilisant uniquement des matériels d'emballage apportés par les familles (cartons, bouteilles en plastique, couvercles, etc.), et en s'exerçant principalement au découpage, au collage et à l'assemblage avec le matériel pédagogique habituel.

Les enregistrements ont été établis de manière à pouvoir identifier les interventions des enfants, et donc à les repérer nominativement (par cochage), l'objectif étant de procéder à une approche principalement qualitative de leurs productions orales en interaction (avec l'adulte ou entre pairs) ${ }^{2}$. Nous n'avons pas recouru, dans ce premier temps de l'analyse, à un traitement prosodique, lequel révélerait plusieurs types de caractéristiques avec une productivité accrue ${ }^{3}$. En revanche, le codage des données (converties au format wav) sous Lexica 5, a permis de disposer d'un corpus linéaire et d'un corpus par participants, de manière à quantifier les mots employés par les enfants et à établir des proportions à l'échelle du groupe.

\section{Description du corpus}

Les aménagements graphiques que nous avons pratiqués sur le corpus linéaire ont été portés à leur minimum. Le moindre ajustement exigeant une justification (Gadet 2003, Dister et Simon 2008), nous avons limité la ponctuation dans les transcriptions, étant donné, notamment, qu'il n'existe pas de correspondances biunivoques entre les manifestations prosodiques, comme les contours intonatifs et les pauses, et la ponctuation graphique (voir aussi Grobet, 1997 ; Shriberg et al. 2000 sur ces derniers points). Dans la mesure, d'autre part, où le cochage s'est révélé suffisant et où l'ensemble est audible, le redressement des productions s'est substitué aux multitranscriptions.

Dans cette première approche, nous avons laissé momentanément de côté les disfluences répétitives se limitant à des supports d'hésitation, et nous

1 L'ATSEM est, dans le système éducatif français, un agent spécialisé des écoles maternelles, qui accompagne les professeurs des écoles dans leurs missions.

2 Le cochage se substitue dans ce cas au support filmique : un tableau nominatif est dressé par groupe et sur plusieurs feuillets, de manière à pointer les interventions (pour certaines concomitantes) des jeunes locuteurs. Une marge est dégagée pour spécifier éventuellement les noms des interlocuteurs de l'enfant qui prend la parole, ou pour indiquer des gestes significatifs.

3 Les principales données primaires pour ce corpus ont été collectées par Julie Damon, professeur des écoles dans l'Académie de Nice et anthropologue de formation. 
n'avons pas indiqué les chevauchements parmi les répliques, lesquels sont d'un apport explicatif minime ici (bien que ces caractéristiques puissent être utiles, évidemment, à d'autres descriptions). Nous donnons, ci-après, un extrait en corpus linéaire non annoté ${ }^{4}$ :

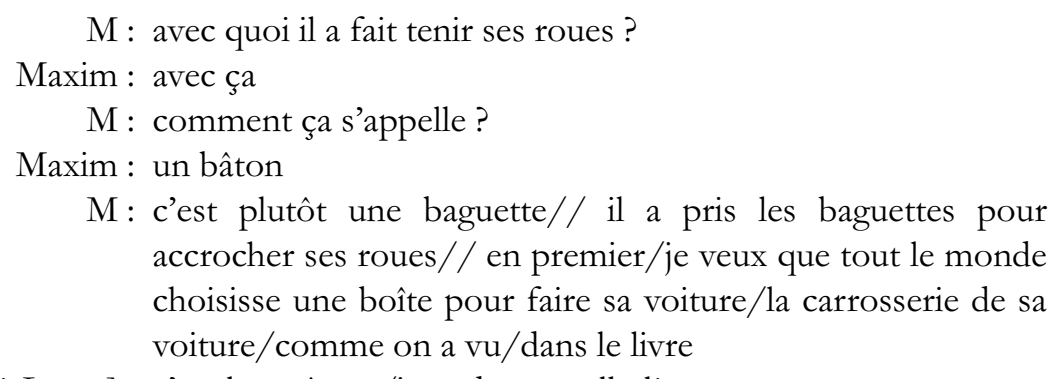

Ambre [à Lucas] : c'est les mêmes/je te donne celle-là

Lucas: je prends les brindilles à la mer et/là les brindilles à la montagne

M : Lucas/tu as choisi ta boîte?

Lucas : je prends celle-là

$\mathrm{M}$ : Anthony/de quoi tu as besoin?

Anthony : deux bâtons

$\mathrm{M}$ : tiens/je te les donne// et essaye de rentrer les roues sur les baguettes/maintenant

Maxim : qu'est-ce que tu fais Kyllian?

Kyllian : bah qu'est-ce que je fais? / paw/paw paw

Ambre : c'est laquelle la plus grande?

Maxim : bah les grandes fusées/ils vont décoller tous ensemble

Ambre : c'est laquelle la plus grande?

$\mathrm{M}$ : où vous en êtes ? / ah je vois un petit Anthony qui travaille très bien/qui a presque compris comment on pouvait faire// je suis très contente Anthony// maintenant regarde cette voiture/comment sont accrochées les roues ? / elles sont toutes du même côté ?

Anthony: non elles sont des deux côtés

4 « $\mathrm{M}$ » renvoie à l'adulte en responsabilité de la classe. Les chevauchements ne sont pas indiqués ici dans la mesure où leur apport explicatif est faible dans cette étude, qui ne place pas du point de vue conversationnel. 


\section{RÉSULTATS DU PREMIER DÉPOUILLEMENT}

Un premier traitement des données a consisté à quantifier les interventions individuelles des enfants et à les rapporter ensuite à celles du groupe, ce qui nous a permis d'établir les proportions dans lesquelles les sujets participent effectivement au dialogue et se manifestent en tant que locuteurs (catégorie codée INTERVENTIONS et abrégée INTERV). Cette variable, qui révèle en partie comment les enfants s'impliquent dans le travail de groupe, a été complétée par le traitement, sous Lexica 5, des contenus eux-mêmes à travers une quantification des mots effectivement employés par les jeunes locuteurs d'une part (qu'ils soient répétés ou non, une catégorie codée MOTS), et, d'autre part, de ce que Lexica 5 étiquette comme "mots uniques ». Cette dernière variable renvoie à la catégorie des mots sans tenir compte du fait qu'ils apparaissent éventuellement plusieurs fois. Un tel codage représente un apport non négligeable dans la mesure où il spécifie non pas les quantités brutes de mots employés (certains, les mêmes, pouvant apparaitre à de multiples occurrences), mais la diversité lexicale des interventions (ce qu'on appelle autrement le " répertoire " de mots produits). Ce facteur, que nous avons dans un premier temps intitulé « mots distincts", sera appelé ici plus commodément ITEMS.

Pour être tout à fait exact, la catégorie des "items » correspond aux éléments du lexique effectivement employés, contrairement à celle des «mots » en données brutes, qui représente la somme des éléments du lexique, dont certains, quelquefois beaucoup, sont récurrents. Indiquons par conséquent que les résultats représentent des proportions : mettons par exemple que, sur 1000 mots employés dans le groupe sur un temps $t$, une élève, Lara, en ait employé 232, le taux reporté dans le tableau sera de 23,2 (pour cent), et ainsi de suite pour les autres. En voici les résultats :

Pour la catégorie des interventions, ces répartitions rejoignent en grande partie les rapprochements et les écarts sur lesquels s'appuient les professionnels de l'enseignement pour déterminer, en plus des données comportementales, quels sont les enfants qui apparaissent plutôt " parleurs » ou au contraire « réticents " (Florin, 1991 ; Corbenois et al., 2000 ; Marcos et al., 2000 ; Mousty $\&$ Leybaert, 2005). Pour autant, là où certaines interventions consistent dans des suites complètement abouties et comportent pour d'aucunes plusieurs phrases, d'autres, en revanche, se limitent à quelques mots et sont beaucoup moins consistantes. Ainsi, concernant les autres variables, là où la première catégorie (MOTS) témoigne de la capacité des enfants proprement à verbaliser, celle des ITEMS est plus significative, en ceci qu'elle détermine la capacité des enfants à employer des mots distincts les uns des autres, et donc à varier leur expression.

Ces tableaux ci-dessus font apparaitre, par exemple, que les productions verbales de Maxim, en 2, occupent 21,83\% des interventions, 26,99\% des mots parmi ceux produits dans le groupe, et que la proportion d'items qu'il emploie correspond à $23,01 \%$ des productions d'ensemble. De même, nous relevons par exemple que Jaed, dans le troisième groupe, s'approprie 15,79\% 


\section{Groupe 1}

\begin{tabular}{lrcr}
\hline & interv & mots & items \\
\hline Apolline & 8,57 & 7,64 & 7,05 \\
\hline Léo & 21,90 & 19,07 & 21,79 \\
\hline Marie Sarah & 11,42 & 9,83 & 7,09 \\
\hline Mathilde & 12,38 & 9,72 & 13,46 \\
\hline Matteo & 20,95 & 23,31 & 23,09 \\
\hline Noémie & 24,78 & 30,43 & 27,52 \\
\hline tot. & 100,00 & 100,00 & 100,00
\end{tabular}

Groupe 2

\begin{tabular}{lrrr}
\hline & interv & mots & items \\
\hline Ambre & 28,73 & 28,92 & 24,60 \\
\hline Antony & 4,59 & 3,30 & 4,76 \\
\hline Kyllian & 16,09 & 14,87 & 15,87 \\
\hline Lucas & 12,64 & 15,15 & 17,48 \\
\hline Maxim & 21,83 & 26,99 & 23,01 \\
\hline Ophélio & 16,12 & 10,77 & 14,28 \\
\hline tot. & 100,00 & 100,00 & 100,00 \\
\hline
\end{tabular}

\section{Groupe 3}

\begin{tabular}{lccc}
\hline & interv & mots & items \\
\hline Alain & 31,57 & 36,37 & 26,82 \\
Jaed & 15,79 & 14,28 & 21,95 \\
\hline Mathis & 7,89 & 10,38 & 12,19 \\
\hline Sarah & 21,05 & 29,87 & 26,82 \\
\hline Shon & 23,70 & 9,10 & 12,22 \\
\hline tot. & 100,00 & 100,00 & 100,00 \\
\hline
\end{tabular}

des interventions et 14,28\% des mots instanciés parmi les enfants, mais que sa capacité à employer des éléments variés du lexique s'élève à $21,95 \%$ par rapport aux capacités du groupe. En général, il existe une corrélation entre les trois types de proportions, ce qui n'est toutefois pas automatique. Ainsi Shon, dans 
le troisième groupe, intervient-il significativement $(23,7 \%)$, mais sa productivité lexicale demeure assez peu élevée par ailleurs ( $9,1 \%$ et 12,22 \%), un schéma qui s'inverse partiellement, par exemple, pour Sarah (avec respectivement 21,05\%, $29,87 \%$ et $26,82 \%$ ).

\section{Discussion}

Si ces résultats renvoient à des caractéristiques opératoires en termes de profils d'apprentissage (les enfants interviennent facilement ou non et verbalisent plus ou moins), leur apport reste intermédiaire en termes de profils d'acquisition. Certes, la première variable contribue à dégager les profils d'enfants plutôt "réticents » ou " parleurs ", mais aussi à mesurer les écarts, éventuellement marqués, qui apparaissent entre eux. De telles données peuvent évidemment influencer directement les dialogues pédagogiques mis en œuvre, ainsi que la formation des groupes elle-même et donc l'organisation de la classe si c'est le cas (Hudelot, 2007), mais leur apport évaluatif reste limité.

Concernant les deux autres types de proportions, celles-ci révèlent qu'en marge des interventions spontanées (autrement dit des " prises de parole »), la parole elle-même est plus ou moins productive d'une part, variée de l'autre. Dans le groupe 1 par exemple, Apolline est à première vue celle qui intervient le moins $(8,57 \%$, alors que les autres résultats vont de $11,42 \%$ à $24,78 \%$ pour le groupe) ; or, si la quantité de mots qu'elle produit (7,64\%) confirme ces faiblesses par rapport aux autres enfants, l'écart ténu entre elle et Marie-Sarah (7,05\% contre $7,09 \%$ ) sur la dernière variable montre que si Apolline produit moins de mots, elle ne les varie pas moins pour autant. Au contraire : comparativement, cette enfant fait montre d'une plus grande capacité que sa camarade à varier les mots qu'elle emploie, même si ces derniers sont en plus faible nombre. Ces disparités apparaissent encore plus nettement avec le sujet Antony dans le groupe 2 : outre les écarts (significatifs) sur la première variable (ce dernier n'occupe que 4,59\% des interventions alors qu'après lui, la quantité la plus basse s'élève à 12,64\%, soit presque trois fois plus), les données démontrent que ce n'est pas parce qu'un enfant produit le moins de mots qu'il est celui dont la capacité à en varier le répertoire est la moindre. L'effort que fournit cet enfant est ainsi en partie confirmé par ses capacités à varier son lexique (4,76\%), tout comme c'est le cas d'Ophélio dans le même groupe (avec respectivement 10,77\% et 14,28\% par rapport aux autres).

L'inverse apparaît aussi, et quelquefois assez clairement. Cette configuration est particulièrement sensible dans le cas d'Alain (en 3), lequel, s'il accapare rien moins que 36,37 \% des mots prédiqués en tout par les participants de son groupe, ne contribue à leur variété que de l'ordre de $26,82 \%$, rejoignant ainsi Sarah $(26,82 \%)$, dont les productions lexicales sont pourtant nettement moindres $(29,87 \%)$.

Nous en retiendrons surtout que si la distinction entre enfants « réticents » et enfants « parleurs » demeure opératoire, il est hâtif d'en conclure que les enfants ne présentent pas d'autres types de disponibilités. Les données démontrent dans 
le même temps que la production de mots n'est pas forcément corrélée à leur variation, et vice versa, et que les profils d'apprentissage (en termes d'appropriation et d'implication) ne coïncident qu'en partie aux profils d'acquisition (en termes de capacités discursives).

\section{RÉSULTATS DU DEUXIÈME DÉPOUILLEMENT}

\section{Présentation des variables}

La deuxième approche du corpus que nous avons pratiquée nous a amenés à en caractériser les éléments prédiqués, de manière à dégager plus spécifiquement, dans les productions, les formes de diversification discursive. Concernant la répartition des éléments qui contribuent tous, dans une certaine mesure, à cette diversification, notre analyse établit une distinction entre d'autres types de variables que celles du premier dépouillement. Parmi elles, nous avons retenu les éléments qui indiquent, suivant les productions, ce dont il est propos (les éléments thématiques : Veneziano \& Hudelot, 2002 ; Morgenstern, 2006), ceux qui prennent appui sur ces derniers et contribuent ainsi à la chaîne discursive (les éléments rhématiques : Mel’čuk, 2001 ; Torterat, 2010b), ceux (moins abondants) qui proprement "balisent " les interventions (les cadratifs : Charolles et al., 2005 ; Asher et al., 2007 ; Luong et al., 2007), et enfin les coordonnants, les subordonnants et leurs assimilés ( $c f$. Rebuschi, 2002), que nous avons regroupés sous la dénomination d'« opérateurs $»^{5}$. Étant donné que le français est une langue "verbo-prédicative », autrement dit s'organise principalement autour du verbe, nous avons pris en compte la contribution, dans les productions orales des jeunes enfants, des verbes en emploi prédicatif, c'est-à-dire, en résumé, des verbes principaux ${ }^{6}$.

Pour ce qui relève de cette catégorie des verbes, nous avons considéré, d'une part, que les verbes ou amas verbaux organisent principalement la structure prédicative des productions verbales (voir là dessus Goldberg, 1995 ; Gerdes \& Kahane, 2006 ; Desclés, 2008), alors que par exemple les opérateurs contribuent à en construire la structure (inter)phrastique, et les cadratifs l'architecture des moments discursifs présents dans les interventions. D’autre part, nous avons porté une attention particulière aux éléments thématiques, qui permettent de poser les objets discursifs dont il est question, et dans une moindre mesure aux

5 Georges Rebuschi concilie, comme d'autres linguistes sensibles à la typologie, coordination et subordination à travers un posé opératoire étendu, qu’il appelle pour sa part la « co-jonction généralisée ». Rappelons par ailleurs que de nombreuses approches grammaticales contemporaines rassemblent les marqueurs des constructions coordinatives et subordinatives à travers la dénomination d'opérateurs.

6 Mettons par exemple la phrase suivante : Mattéo a pris ma roue et il a voulu la casser. Dans cette suite, les formes composées du verbe a pris et a voulu sont des verbes principaux en ceci qu'ils organisent les éléments qui sont sous leur attraction (ainsi les sujets Mattéo et $i$, les compléments ma roue et la casser). Dans cette vue, casser, même s'il conserve la possibilité d'avoir lui-même un complément (la), n'en demeure pas moins l'un des arguments du verbe principal vouloir et, à ce titre, est moins prédicatif que lui. 
éléments rhématiques, lesquels apportent des informations à propos des éléments thématiques (que ces derniers soient instanciés ou non). Nous suivons en cela Mel'čuk (op. cit.), qui, pour ce qui concerne la «thématicité », établit que le thème correspond à la partie de la prédication qui présente ce à propos de quoi les informations sont données ${ }^{7}$.

\section{Codage des données}

Cette répartition, parmi les éléments instanciés dans les productions verbales des enfants, nous a paru suffisamment significative pour l'appliquer sans apport logiciel à l'ensemble du corpus, aucun programme n'existant encore pour ce type d'analyse. Cela a impliqué une réédition du matériau, comme l'exemplifient les extraits que nous reproduisons infra. Par commodité, nous indiquons en italiques les verbes et amas verbaux, en gras les opérateurs (pour la plupart des coordonnants et des subordonnants), en majuscules les ÉLÉMENTS THÉMATIQUES, en petites majuscules les ÉLÉMENTS RHÉMATIQUES, et nous soulignons enfin les cadratifs. Les barres obliques $(/, / /$ ) renvoient aux marques pausales ou aux frontières prosodiques, et les barres verticales $(\mid$ ) spécifient les séparateurs syntaxiques qui font des éléments précédent et subséquent, au moment de leur quantification, des informations disjointes.

Ce nouveau codage, pratiqué donc manuellement, permet un étiquetage plus fin des mêmes données que le premier dépouillement, tout en prenant en considération la dimension pragmatique des productions interlocutives, comme nous le reportons ci-après d'abord dans sa configuration interlocutive (extrait 1), puis en indiquant ce que donne l'étiquetage uniquement par participants (extraits 2 et 3):

Extrait 1 (les interventions de M, l'adulte, n'étant pas codées, elles apparaissent intégralement en italiques)

Léo : il_ NOUS faut DES BÂTONS

Mattéo : DES PIQUES

$\mathrm{M}$ : il nous faut des piques? / il nous en faut combien Mathilde?

Mathilde: UN/DEUX

M : deux ? / alors je te donne deux bâtons// tu as pris une boîte Léo ? / choisis ta boîte

Noémie : CELLE-LÀ

Léo : OUI | CELLE-LÀ

$\mathrm{M}$ : alors je vais te donner des bâtons plus courts/sinon ça va trop dépasser// prends ceux-là// Mathilde/tu as choisi une boîte?

Mathilde: LA BOITE DES BISCUITS

$\mathrm{M}$ : très bien

7 Nous intégrons pour notre part les « spécificateurs », dans la terminologie de Mel'čuk, à ce que nous appelons les cadratifs et certains opérateurs. 
Mattéo : moi c'est LA BOÎTE D'EUUFS// ça c'est UNE BOÎTE D'CEUfS Extrait 2

Mattéo : après JE TE rends/| d'accord?

Mattéo : moi ÇA | ME fait même pas MAL

Mattéo : attendez.JE vais_PLUTÔT mettre COMME ÇA

Mattéo : DES PIQUES

Mattéo : DES ROUES

Mattéo : des roues/il manque DES ROUES

Mattéo : fais voir Noémie

Mattéo : heu/QUATRE

Mattéo: J'ai réussi | à faire $\mathrm{UN}$ TROU | LÀ

Mattéo : JE veux mettre LES BAGUETTES et J'arrive pas

Mattéo : Julie TU peux_ M'aider?

Mattéo : LÀ

Mattéo : moi c'est LA BOÎTE D'EUfS// ça c'est UNE BOÎTE D'CEUfS

Mattéo : moi J'arrive pas |à_ LE faire

Mattéo : moi JE L'ai fait

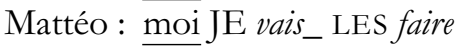

Mattéo : OUI c'est TOUT

Mattéo : OUI

Mattéo : parce que// (parce que) les trous ILS veulent pas | SE faire

Mattéo: QUATRE

Mattéo : TU peux_M'aider | à faire UN TROU ?

Mattéo : y a pas DES ROUES

Extrait 3

Ophélio : accrocher LES ROUES ?

Ophélio : COMME ÇA

Ophélio : DEUX

Ophélio : ENCORE UN

Ophélio : et | après JE mets LES ROUES

Ophélio: Heu QUATRE

Ophélio : il manque DES ROUES

Ophélio : JE prends DES ROUES

Ophélio: JE veux UN TROU | LÀ Julie

Ophélio: DES ROUES

Ophélio: NON// ICI

Ophélio: OUI

Ophélio: OUI/| EN FACE

Ophélio : regarde 


\section{RÉSULTATS}

La prise en compte des informations contextuelles à l'aide des données primaires a été assistée par d'autres indicateurs propres à l'oral, principalement suprasegmentaux (contours intonatifs, pauses, intensifications). Par ailleurs mais nous ne pouvons ici reproduire l'ensemble du corpus -, les données pragmatiques (reprises dialogales, enchaînements, etc.), à l'exception des verbes et des opérateurs intraphrastiques, ont été déterminantes pour une partie des cas concernant les autres variables. En revanche, les regroupements effectués par Lexica, que nous avons sollicité dans un premier temps, procèdent en partie par assemblages fortuits et ne distinguent pas certains (pseudo)-homographes (comme la et là par exemple) sur les données lexicales, ce qui oblige donc à opérer, comme nous l'avons expliqué, un étiquetage spécifique sur l'ensemble du corpus.

Indiquons que ce codage implique de spécifier les constructions verbales elles-mêmes dans les productions de manière à pratiquer des regroupements plus locaux. Ainsi, les verbes accompagnés d'un marqueur de négation ont-ils été envisagés comme des verbes négativés (tout comme ils peuvent être "amassés » ou passivés ${ }^{8}$. D'autre part, nous n'avons comptabilisé, dans les proportions ci-dessous reportées, ni les opérateurs suspensifs du type beu (lesquels marquent des opérations diverses et se caractérisent surtout par l'intermédiaire des contours prosodiques), ni les interjections du type aye, ab ou iliou (lesquelles peuvent être thématiques, rhématiques, voire cadratives : Wilkins 1992, Cuenca et Hilferty 1999). Avec une consistance phonologique similaire cependant, nous avons par exemple conservé un ata produit par un élève, mot que nous avons considéré non pas comme un cadratif (Balthasar et al. 2003), mais comme un attends d'action, autrement dit comme un verbe?

Nous reviendrons sur les marges d'erreur possibles, lesquelles ne peuvent être complètement réduites vu l'ensemble des variables à catégoriser, mais nous donnerons d'abord les résultats, là encore en termes de proportions, du présent traitement :

Ces résultats font ainsi apparaitre qu'Alain, dans le groupe 3, s'approprie $33,2 \%$ des verbes et amas verbaux du groupe, $43,82 \%$ des éléments thématiques, mais seulement $6,31 \%$ des éléments rhématiques. De même dans le groupe 2, Lucas n'emploie que $6,34 \%$ des cadratifs utilisés par les participants, mais ses productions oscillent par ailleurs entre 12,07 et $22,86 \%$ des emplois recensés. Ces données confirment qu'une analyse des emplois et des réemplois des marqueurs discursifs ne peut en aucun cas laisser présumer des résultats que produiraient les autres variables. Une certaine stabilité existe néanmoins dans certains cas : ainsi les proportions sont assez stables chez Maxim notamment, dans le groupe 2 (où sa productivité oscille entre $24,56 \%$ et $35,89 \%$ quelles

8 Verbe négativé : [il] (ne) regarde pas; amassé : [il] va regarder; passivé : [il] a été regardé.

9 Attends (verbalisé ata ici par l'enfant) n'est pas un simple marqueur discursif de suspension du dire, mais signifie effectivement une injonction de faire, matérialisable et concrète. 
Groupe 1

\begin{tabular}{lrrrrr}
\hline & verbes & them & rhem & cadr & op \\
\hline Apolline & 9,23 & 12,48 & 4,92 & 11,06 & 4,17 \\
\hline Léo & 18,84 & 15,67 & 20,12 & 14,99 & 13,08 \\
\hline Marie Sarah & 7,21 & 7,63 & 10,77 & 19,11 & 0,00 \\
\hline Mathilde & 9,88 & 12,95 & 11,19 & 8,56 & 3,60 \\
\hline Matteo & 25,03 & 21,09 & 23,36 & 22,81 & 7,79 \\
\hline Noémie & 29,81 & 30,18 & 29,64 & 23,47 & 71,36 \\
\hline tot. & 100,00 & 100,00 & 100,00 & 100,00 & 100,00
\end{tabular}

Groupe 2

\begin{tabular}{lrrrrr}
\hline & verbes & them & rhem & cadr & op \\
\hline Ambre & 31,25 & 27,75 & 23,37 & 40,98 & 34,09 \\
\hline Antony & 1,20 & 2,18 & 5,66 & 0,00 & 0,00 \\
\hline Kyllian & 15,82 & 16,24 & 9,62 & 17,81 & 11,91 \\
\hline Lucas & 14,96 & 22,86 & 15,19 & 6,34 & 12,07 \\
\hline Maxim & 28,49 & 24,56 & 24,73 & 29,20 & 35,89 \\
\hline Ophélio & 8,28 & 6,41 & 21,43 & 5,67 & 6,04 \\
\hline tot. & 100,00 & 100,00 & 100,00 & 100,00 & 100,00 \\
\hline
\end{tabular}

Groupe 3

\begin{tabular}{lrrrrr}
\hline & verbes & them & rhem & cadr & op \\
\hline Alain & 33,20 & 43,82 & 6,31 & 61,95 & 0,00 \\
\hline Jaed & 8,76 & 14,19 & 24,57 & 13,02 & 0,00 \\
\hline Mathis & 14,89 & 13,80 & 18,83 & 0,00 & 0,00 \\
\hline Sarah & 28,05 & 13,97 & 44,22 & 25,03 & 0,00 \\
\hline Shon & 15,10 & 14,22 & 6,07 & 0,00 & 0,00 \\
\hline tot. & 100,00 & 100,00 & 100,00 & 100,00 & 100,00 \\
\hline
\end{tabular}

que soient les variables retenues). Pour autant, s'il existe par exemple une certaine uniformité des capacités de Noémie, dans le groupe 1, pour ce qui relève des quatre premières variables (avec respectivement 29,81 \%;30,18 \% ; $29,64 \%$ et $23,47 \%$ ), cette tendance s'accroît significativement dans ce domaine 
des opérateurs, où sa contribution est maximale (avec 71,36\%), une telle contribution témoignant du fait qu'elle a une capacité bien plus marquée que les autres dans le domaine de la construction phrastique. L'uniformité n'apparaît qu'en partie, pour prendre un autre exemple, avec Sarah (dans le groupe 3), laquelle, si elle contribue significativement à la production des verbes instanciés dans son groupe $(28,05 \%)$, ainsi que des cadratifs $(25,03 \%)$ et des éléments rhématiques $(44,22 \%)$, participe dans une moindre mesure à la production des éléments thématiques (avec 13,97\% proportionnellement au groupe).

\section{Première discussion : analyse critique des marges d'erreurs}

Ce modèle d'analyse n'est pas sans présenter une marge d'erreur qu'il convient de soumettre à d'éventuelles critiques. Effectivement, une proportion, du reste assez mesurée, de « cas douteux » ont persisté, ces derniers occupant de $3 \%$ à $6 \%$ environ des éléments collectés suivant les variables.

\section{Concernant les verbes et amas verbaux}

1. Ambre : accrocher LES ROUES

2. Ambre: il faut pas| LES abîmer

3. Shon : ata

En 1, en marge du fait qu'accrocher présente d'indéniables caractéristiques verbales (comme la capacité d'avoir un complément d'objet en transitivité directe), la question se pose de savoir s'il ne participe pas significativement à la thématicité, autrement dit intervient dans la production de l'enfant comme élément thématique (ce à quoi nous n'avons pas souscrit). Le cas de 2 pose de son côté une difficulté relative à la quantification des éléments : convient-il, ici, de distinguer la construction unipersonnelle il fautpas du complément abîmer, sous prétexte que ce dernier constitue, avec le clitique les qui le précède, un syntagme verbal à sujet indéterminé mais dont la paraphrase avec une conjonction (qu'on les abîme) suppose une construction subordinative non marquée ? Convient-il, au contraire, d'intégrer ce dernier dans une locution verbale ? Si nous avons pour notre part choisi la première solution, du fait de la construction transitive avec falloir et de l'intercalation du pronom rhématique les, cela reste sujet à discussion. Le cas de 3, enfin, pose un problème d'intelligibilité, et donc de qualification, même si nous avons estimé qu'il s'agit d'une forme verbale altérée $(M$ : on va essayer/avec une roue en plus alors Alain// tiens/Sarah/tu es sûre que t'en veux qu'une toi aussi ? - Shon : ata/- $M$ : très bien/regardez. Shon// il a réussi à enfiler une roue sur le bâtonnet-Sarah : il faut/ $/$.

\section{Les éléments thématiques}

Ici, d'autres profils de difficultés apparaissent, dont nous donnons ci-après cinq illustrations :

\section{Léo : il_ NOUS faut DES BÂTONS}




\section{Noémie : et moi CETTE BOÎTE}

3. Noémie : pourquoi ELLE a pris UNE ROUE QUI marche pas TRÈS BIEN ?

4. Alain : MOI veux faire

5. Ambre : c'est LAQUELLE LA PLUS GRANDE?

Dans 1, l'intercalation d'un pronom rhématique dans la construction unipersonnelle, avec la correction que cela suppose chez l'enfant, peut inciter à inclure le clitique il comme élément thématique, ce que nous n'avons pas pu trancher (nous l'avons donc intégré dans la variable «verbes » et regroupé avec falloir). Dans 2, nous avons estimé que moi apparaît plutôt comme cadratif ici, du fait d'une construction verbale transitive elliptique (incomplète), bien qu'il soit possible de l'envisager comme l'élément thématique d'une phrase averbale, ce à quoi nous nous sommes résolu pour le moi de 4 ( $c f$. Apothéloz, 1997), où la phrase est verbale. L'énoncé 3, de son côté, exemplifie le fait que l'opérateur qui (subordonnant) constitue aussi un élément thématique et peut être reconnu comme tel, cette question posant indirectement celle, par ailleurs, du poids structural que l'on peut assigner à un mot qui accomplit plusieurs opérations en même temps (Geeraerts, 1991). Or, le même type de question se pose vis-à-vis de laquelle, en 5, qui, en construction attributive directe, fait l'objet d'une extraction dans un cadre haplologique (non-répétition du support) et elliptique (c'est laquelle (qui $i_{\text {hapl }}$ ) (estell) la plus grande), ce qui fait simultanément de cet opérateur, selon nous, un élément thématique plutôt que rhématique.

\section{Les éléments rhématiques}

Concernant ce type d'éléments, ce sont surtout les constructions verbales et l'ordre des mots qui permettent de les catégoriser, comme le montrent les cas reportés ci-après :

1. Léo : J'ai compris/il faut EN mettre| DE L'AUTRE CÔTÉ

2. Léo : LÀ/| AU BORD.

3. Mathilde : moi J'ai UNE ROUE QUI marche pas TRÈS BIEN

4. Mattéo : attendezJE vais_PLUTÔT mettre| COMME ÇA

5. Noémie : et c'est quoi ÇA/| CET OUTIL ? / C'est COMME UN BOUCHON

Le pronom en, dans 1, est-il intégré dans une locution verbale ou se comporte-t-il plutôt comme un complément conjoint, apparaissant ainsi comme élément rhématique au même titre que le complément adjoint de l'autre côté ? C'est ce que nous supposons, d'autant que ce cas est plus facile à trancher que dans 5 , où les deux attributs ça et cet outil, qui sont coréférents, apparaissent dans la même chaîne discursive que la proforme quoi et le ce élidé sujet du verbe être. Pour 5 encore et concernant les deuxième et troisième constituants attributifs (ça/cet outi), nous avons estimé qu'il convient de les distinguer, en marge de la construction en apposition, étant donné que le fait de les fusionner en un seul élément rhématique ne permettrait pas de rendre compte du caractère reformulatif du deuxième élément. 
D'autres critères ont été relevés dans ce domaine, comme en témoignent les caractérisations de l'adverbe là et du syntagme au bord dans 2 : le premier peut-il être envisagé comme un cadratif, ce que semblent confirmer les emplois multiples qui en sont effectués par ailleurs dans les productions verbales des enfants (en position préverbale devant tous types d'éléments), tandis qu'au bord interviendrait plutôt comme un circonstant (Ho-Dac \& Frérot, 2004) ? Ce sont ici les éléments cotextuels qui nous permettent de trancher, Léo répondant dans ce cas à la question suivante de $\mathrm{M}$ : je le fais où le deuxième trou?

Par ailleurs, et c'est là une question importante pour le traitement des formes de diversification discursive, ces éléments témoignent du fait que les marqueurs spécifiquement discursifs utilisés par les enfants ont une affinité particulière avec les positions qui précèdent le verbe, dans la mesure où la plupart des marqueurs concernés intègrent ce qui peut être appelé, suivant les approches linguistiques représentées, un "préfixe » (Blanche-Benveniste, 2003) ou un " préambule thématique » (Morel \& Danon-Boileau, 1998), lesquels occupent une position stratégique d'un point de vue informationnel et mémoriel, mais aussi participent de ce que François, Hudelot et Sabeau-Jouannet (1985) appellent une « conduite de monstration » (60).

Le cas du SN une roue de 3 est plus difficile à traiter : intégré dans une construction transitive spécifique et faisant l'objet d'une extraction avec subordination (j'ai $S N_{-}$qui $V B$ ), et formant par ailleurs l'antécédent d'un pronom sujet qui intervient, dans la subordonnée, comme un élément focal, il s'avère également focalisé par la construction qui l'encadre. Nous l'avons compté comme élément rhématique, bien qu'il puisse être envisagé comme thématique à quelques égards. Quant au cas de 4, celui-ci pose des difficultés plus « classiques ", si l'on nous passe l'expression, en ceci que plutôt constitue un élément rhématique d'une portée si faible qu'il est possible de l'intégrer dans l'amas verbal.

\section{Les cadratifs}

Dans ce domaine, l'hésitation porte surtout sur le fait d'envisager les éléments comme tels ou comme des circonstants (rhématiques), ce qui est là aussi très prévisible :

1. Mathilde : voilà

2. Alain : JE veux là

3. Jaed : yo YA BOU

Comme nous l'avons évoqué supra, la plupart des cadratifs occupent une position qui précède le verbe dans les dialogues enfantins, à moins qu'ils ne soient rejetés (extraposés), comme c'est le cas à notre sens du là de 2 : dans ce contexte, il ne circonstancie pas le verbe vouloir, mais indique, chez l'enfant, que c'est en tant que locuteur qu'il veut rester au moment où l'intervenante « $\mathrm{M}$ » lui suggère de passer dans le coin bibliothèque. L'adverbe renvoie ici, selon nous, à un moi/là, autrement dit réaffirme l'enfant en tant que participant au groupe (comme c'est le cas, vraisemblablement, du yo de 3). De son côté, le voilà de 1 pourrait à première vue renvoyer à un élément rhématique dans le 
cadre d'une prédication non verbale, pour ainsi dire comme un rhème, mais là encore, le contexte témoigne du fait qu'il positionne davantage l'enfant dans un contexte interpersonnel et discursif qu'il n'apporte une information sur un élément thématique précédemment instancié, comme on le voit ci-après (en corpus linéaire) :

$\mathrm{M}$ : qu'est-ce qui est trop haut?

Noémie : LES ROUES

Marie-Sarah : AH OUI

$\mathrm{M}$ : ah oui/on dirait// alors/est-ce que tu veux que je fasse un trou à un autre endroit?

Noémie : OUI il faut FAIRE | UN TROU | LÀ | TOUT EN BAS

Mathilde : voilà

\section{Les opérateurs}

Ces derniers peuvent eux aussi susciter quelques hésitations :

1. Noémie : OUI ELLE roule/mais JE ne LA touche pas sinon LES ROUES tombent

2. Maxim : QUATRE/| COMME QUATRE ROUES

Le cas 1 comporte un opérateur ne qui n'est pas ordinaire dans les productions verbales des enfants, ce qui a justifié, de notre point de vue, le fait de l'extraire d'un syntagme verbal où nous n'avons pas comptabilisé l'autre opérateur de négation (tout ensemble [VB-NEG] a été considéré non pas d'abord comme un verbe accompagné d'un adverbe de négation, mais comme un verbe négativé, formant ainsi une unité à proprement parler unique). L'énoncé 1 contient par ailleurs une préposition sinon que nous avons comptée parmi les opérateurs, dans la mesure où elle est ici assimilable à un subordonnant, une caractéristique que nous avons appliquée également au comme de 2 .

Ces marges d'erreur impliquent que l'étiquetage se fasse manuellement sur l'ensemble, étant donné la sensibilité au contexte de la catégorisation des éléments ( $c f$. Auer, 2002). Pour autant, la productivité de cette approche analytique compense, à notre sens, le coût que représente son opérationalisation.

\section{DEUXIÈME DISCUSSION : CE QUE RÉVÈLENT LES PROPORTIONS}

Les capacités que révèlent les quantités dégagées permettent très concrètement d'établir des liens directs, de notre point de vue, entre profils d'apprentissage et profils d'acquisition.

Pour ce qui relève de l'apprentissage, ces résultats témoignent de variations interindividuelles contrastées dans les domaines de l'intégration du groupe, de l'implication dans la conduite pédagogique ainsi que dans la prise en compte d'autrui (Torterat, 2009, 2010a). Pour ce qui concerne l'acquisition, les données 
révèlent des capacités là aussi contrastées mais sur des plans différents, dont l'un des plus significatifs (dans la présente analyse), est précisément celui de la diversification discursive. Or, cela suppose que nous abordions les tableaux sous plusieurs angles.

Si on ne peut dire que les enfants qui participent abondamment à la variable des «éléments thématiques » sont ceux qui fournissent le plus couramment les apports d'information à partir desquels se tient le dialogue entre les locuteurs, ce qui n'est vrai qu'en partie, on peut avancer sans difficulté qu'une telle productivité témoigne d'un effort incontestable pour se faire comprendre, ainsi que d'une capacité concrète dans le domaine des repères d'expression. À l'inverse, une productivité accrue dans la variable rhématique peut indiquer chez l'enfant, outre des facultés de compréhension, à la fois une implication dans le dialogue pédagogique et, en termes d'acquisition, une capacité à compléter, à caractériser et à modifier les apports thématiques sur lesquels il prend appui. Le sujet Alain, en 3, en apporte une démonstration directe, car ses capacités à produire des éléments thématiques s'élèvent à 43,82 \%, alors qu'il contribue très peu, par ailleurs, à la variable rhématique (pour seulement 6,31\%). Ces contrastes apparaissent alors même que ses phrases ne sont pas forcément sommaires (il emploie à lui seul 33,2 \% des verbes et amas verbaux du groupe) et qu'il s'attache dans le même temps à organiser plus ostensiblement ses interventions (avec rien moins que $61,95 \%$ des cadratifs). Si on lit obliquement les données du tableau, on remarque ainsi que la disponibilité de Shon pour les éléments rhématiques (de $6,07 \%$ ) est assez proche de celle d'Alain, qui produit pourtant plus de trois fois plus d'éléments thématiques $(43,82 \%$ donc, contre 14,22\% pour l'autre élève, chez lequel la corrélation qui s'établit entre les verbes et les thèmes - avec respectivement 15,1 et $14,22 \%$ - est beaucoup plus marquée).

Un autre type de disponibilité apparaît évidemment, à l'inverse, quand les enfants s'appuient indéniablement sur ce que disent les autres pour compléter, et modifier éventuellement, ce qui fait l'objet du dialogue. Ainsi Antony, en 2, présente-t-il des difficultés à formuler des phrases verbales (avec 1,2\%), de même qu'à indiquer ce dont il parle $(2,18 \%$ des thèmes). En revanche, sa participation s'accroît dès lors que les éléments thématiques ont été instanciés, car même si elle reste modeste, sa production rhématique s'élève à 5,66 \%. C'est ce qui se produit également, par exemple, pour Marie-Sarah, en 1, laquelle contribue faiblement à la production des éléments thématiques $(7,63 \%)$ de même qu'aux verbes et amas verbaux $(7,21 \%)$, mais s'implique ouvertement dans le dialogue à la fois en parlant à propos de ce qui est dit (avec 10,77\% des rhèmes) et en tâchant d'organiser au mieux son intervention afin de se faire comprendre (avec 19,11\% des cadratifs).

À ce propos précisément, ce qui rapproche les cadratifs des opérateurs consiste dans la possibilité que nous fournissent les résultats d'envisager concrètement dans quelle mesure les enfants tâchent de se faire comprendre et de structurer leurs interventions. Une lecture verticale des résultats dans cette perspective permet assez vite de mesurer les écarts entre les productions. Si 
nous prenons par exemple le groupe 1, la capacité d'employer des opérateurs est quasiment monopolisée par une élève, Noémie (71,36 \%), qui n’est rejointe que bien après par Léo (13,08 \%) dans sa démarche. Ces contrastes sont beaucoup moins élevés dans le groupe 2, où les quantités varient de 0 à 35,89 \% mais où trois effectifs de capacités apparaissent clairement (autour respectivement de 0\%-6 \%, de $11 \%-12 \%$ et de $34 \%-35 \%$ ). Pour les cadratifs, qui témoignent de la possibilité de structurer son propos dans son ensemble, mais aussi, proprement, d'organiser son dire, les résultats montrent que cet effort compense dans certains cas des faiblesses par ailleurs. Nous avons parlé de Marie-Sarah, mais nous pourrions tirer des conclusions similaires pour Ambre (en 2), qui encadre plus qualitativement ses productions discursives (40,28\%) qu'elle ne produit de verbes $(31,25 \%)$ ou d'éléments rhématiques (23,37\%), ainsi que pour Sarah (en 3), qui compense, avec $25,03 \%$ des cadratifs de son groupe, sa faible implication dans les éléments thématiques $(13,97 \%)$.

Nous pourrions multiplier les commentaires sur les écarts et les rapprochements entre les productions locutoriales des enfants. Nous retiendrons surtout de ce deuxième traitement, pratiqué sur notre corpus de référence (dont la représentativité doit être évidemment confrontée à de plus grands corpus), que ces résultats nous conduisent à relativiser les jugements sur ce qui fait la productivité des interventions verbales des jeunes enfants, mais aussi sur ce qu'est véritablement un enfant " parleur».

Des tendances apparaissent néanmoins : selon nous, l'emploi abondant d'éléments rhématiques témoigne d'une intégration discursive effective, alors qu'une production accrue d'éléments thématiques révèle une capacité d'instanciation et pour ainsi dire d'effectuation du propos. Pour leur part, les cadratifs et les opérateurs démontrent à la fois une prise en compte d'autrui plus aboutie et, éventuellement, une concrète facilité à faire preuve d'une démarche discursive autonome. Comme nous avons pu l'observer par ailleurs, ces tendances se remarquent également quand l'apprentissage de la parole coïncide avec celui des tout premiers écrits (Torterat, 2009, 2011, à paraître). En revanche et dans tous les cas, nous serons beaucoup plus mesurés sur l'apport de la catégorie verbe, en ceci que les verbes ont un poids prédicatif très variable dans les productions, mais aussi en ceci qu'ils intègrent par ailleurs des éléments thématiques ou rhématiques (comme c'est le cas des compléments infinitivaux par exemple), sans qu'on ne puisse en déduire autre chose qu'une facilité à se (re)formuler.

\section{Quelques perspectives pour l'analyse}

Si un traitement en données strictement quantitatives (interventions, mots et items) présente un caractère trivial, il est toutefois en partie conforté par celui des formes de diversification discursive, dont la prise en compte permet, en revanche, de corriger les apories des quantifications brutes avec les possibilités suivantes :

- le deuxième type de traitement fournit un cadre méthodologique possible pour l'analyse de corpus longitudinaux ; 
- ses informations quantifient les corrélations existant entre diversification syntaxique et structuration discursive ;

- les proportions qu'il révèle nous incitent à caractériser avec plus d'exactitude les conduites discursives présentes parmi les verbalisations enfantines.

De notre point de vue, ce type d'approche complète, tout en les contrariant en partie, certains traitements de corpus oraux certes facilement paramétrables, mais aux conclusions peu garanties. Ces faiblesses sont tout particulièrement le fait d'approches qui prennent en compte, comme variables de production, la catégorie des noms, celle des déterminants ou celle des verbes, pour en dresser ensuite des profils de développement de l'enfant. Selon nous, établir des corrélations entre les profils d'acquisition et les manières dont les jeunes enfants produisent soit des noms, soit des verbes (ou des mots de toute autre catégorie grammaticale), conduit à une forme d'aberration épistémologique. Le fait de placer l'analyse à l'aune de telles catégories, à notre sens, fragilise l'étude des acquisitions proprement linguistiques, lesquelles ne peuvent être réductibles à des paradigmes qui relèvent de la grammaire, avec les jugements et les présuppositions qu'elle implique. Cette conformisation est d'autant plus dommageable qu'elle ne résiste pas au fait de transcatégorialité d'une part, et à celui de polyopération de l'autre. Par exemple, il est avéré que, rien que pour la catégorie verbe, les formes non fléchies démontrent que leur existence en tant que verbes dépend de l'emploi qui en est effectué en discours. Un infinitif est de ce fait facilement nominalisable, un participe est au moins en partie (et très variablement) adjectivé, et cela sans parler de l'existence des déverbaux, des conversions possibles ou des locutions (qui apparaissent comme un tout). De même, un déterminant accomplit de facto plusieurs opérations simultanément : dans le même temps qu'il singularise ou qu'il généralise un support nominal par exemple, il le quantifie ou le caractérise éventuellement, de même qu'il contribue, dans bien des cas, à inscrire l'élément déterminé dans une chaîne d'accord. Or, que déduire d'une approche qui relèverait tous les déterminants sans évaluer leur contribution, là aussi variable, à des caractéristiques qui se combinent en discours?

Une approche discursive permet, de notre point de vue, d'éviter les automatismes qui soumettent les acquisitions linguistiques aux catégorisations préconstruites de la grammaire. Si l'on en suit notre analyse, il apparaît par exemple qu'une productivité accrue dans la variable thématique renvoie aux capacités d'instanciation et de topicalisation, tandis que celle de la variable rhématique correspond davantage à une faculté d'intégration dialogale et de complémentation. D'autre part, on peut inférer dans un premier temps que les cadratifs témoignent d'une faculté d'organiser le propos dans son entièreté, alors que les opérateurs démontrent une capacité non pas de construire l'entièreté du propos, mais de combiner des éléments discursifs qui le constituent, que ces derniers soient envisagés à l'échelle de groupes intonatifs, de périodes ou de phrases.

Dans tous les cas, l'approche des corpus oraux collectés dans un cadre interlocutif contribue à dégager certains questionnements propres à la 
construction des productions verbales, comme la transitivité, la négation et l'extraction. Simultanément, elle nous invite à relever des informations relevant de la structuration discursive, comme la thématicité, mais aussi l'encadrement discursif. Le traitement et l'analyse des données révèlent alors des récurrences d'éléments spécifiques en production, ce qui, en linguistique de l'acquisition tout comme en contexte d'apprentissage, peut représenter un apport non négligeable en termes d'individualisation et de prise en compte de la diversité des enfants.

\section{RÉFÉRENCES BIBLIOGRAPHIQUES}

Apothéloz, D. (1997). «Les Dislocations à gauche et à droite dans la construction des schématisations ", in A. Berrendonner et D. Miéville (éds.), Logique, Discours et Pensée. Mélanges offerts à Jean-Blaise Grize. Bern : Lang, 183-217.

Asher, N., Prevot, L., Vieu, \& L. (2007). «Setting the background in discourse », Discours 1 [Online]. URL : http://discours.revues.org/index301.html.

Auer, P. (2002). " Projection in interaction and Projection in grammar ». Interaction and Linguistic Structure, 33, 1-43.

Balthasar, L., Bruxelles, S., Mondada, L, \& Traverso, V. (2003). «Attends ça fait travailler le cerveau: usages et tendances à la grammaticalisation de attends en français parlé en interaction ", Linguistique de Corpus, $36^{\mathrm{e}}$ colloque de la Societas Linguistica Europea. Lyon, 4-7.

Bassano, D. (2005). " Production naturelle précoce et acquisition du langage : l'exemple du développement des noms ", in M. Savelli (éd.), Lidil (Corpus oraux et diversité des approches) 31, 61-84.

Bassano, D., Maillochon, I., \& Eme, E. (1998). "Developmental changes and variability in early lexicon : a study of french children's naturalistic productions ", Journal of Child Language, 25, 493-531.

Blanche-Benveniste, C. (2003). Approches de la langue parlée en français. Paris : Ophrys.

Boysson-Bardies (de), B., \& Vihman, M. M. (1991). "Adaptation to language: Evidence from babbling and first words in four languages", Language, 67, 297-319.

Charolles, M., Le Draoulec, A., Pery-Woodley, M. P., \& Sarda, L. (2005). “Temporal and spatial dimensions of discourse organisation", Journal of French Language Studies, 15(2), 203-218.

Corbenois, M., Devanne, B., Dupuy, E., \& Martel, M. (2000). Apprentissage de la langue et conduites culturelles. Paris : Armand Colin.

Cuenca, M. J., \& Hilferty, J.J. (1999). Introducción a la linguistica cognitiva. Barcelona : Editorial Ariel.

Desclés, J. P. (2008). "Opérations de prédication et de détermination », Lidil 37, 61-98.

Dister, A., \& Simon, A. C. (2008). «La transcription synchronisée des corpus oraux. Un aller-retour entre théorie, méthodologie et traitement informatisé », Arena Romanistica, 1(1), 54-79.

Florin, A. (1991). Pratiques de langage à l'école maternelle et prédiction de la réussite scolaire. Paris : PUF. 
François, F., Hudelot, Ch., \& Sabeau-Jouannet, E. (1985). Conduites linguistiques chez.le jeune enfant. Paris : PUF.

Gadet, F. (2003). La Variation sociale en français. Paris : Ophrys.

Geeraerts, D. (1991). " La Grammaire cognitive et l'histoire de la sémantique lexicale », Communications, 53, 17-50.

Gerdes, K., \& Kahane, S. (2006). "L'amas verbal au cœur d'une modélisation topologique de l'ordre des mots », Linguisticae Investigationes, 29(1), 75-89.

Goldberg, A. (1995). Constructions. A Construction Grammar Approach to Argument Structure. Chicago: Chicago University Press.

Grobet, A. (1997). «La Ponctuation prosodique dans les dimensions périodique et informationnelle du discours », Cabiers de Linguistique française, 19, 83-123.

Ho-Dac, L-M., \& Frérot, C. (2004). « Approche discursive et approche syntaxique des circonstants en corpus ». Journées ATALA, La RochelleFrance [consulté le 5 octobre 2009] : http://w3.erss.univ-tlse2.fr:8080/index. jsp?perso $=$ hodac\&subURL=pageperso_fichiers $/$ HoDac-Frerot-atala.pdf.

Hudelot, Ch. (2007). «The Use of a Functional Dialogic Model of Verbal Interaction to Compare how Day-care Workers and Teachers Scaffold 3-year-old Children », in L. Berlin (éd.), Theorical Approaches to Dialogue Analysis. Tübingen: Niemeyer, 215-227.

Kail, M., \& Bassano, D. (2000). « Méthodes d'investigation et démarches heuristiques ", in M. Kail \& M. Fayol (éds.), L'Acquisition du langage (vol. 1 : Le Langage en émergence). Paris: PUF, 29-60.

Luong, X., Juillard, M., Mellet, S., \& Longrée, D. (2007). “The Concept of Text Topology. Some Applications to Verb-Form Distributions in Language Corpora", Literary and Linguistic Computing, 22(2), 167-186.

Marcos, H., Salazar Orvig, A., Bernicot, J., Guidetti, M, Hudelot, Ch., \& Préneron, C. (2000). " Le développement du langage et la communication : l'influence du mode d'accueil chez les enfants de deux et trois ans ", Recherches et prévisions, 62, $57-70$.

Martel, K., dir. (2009). Enfance, 61(3), Quand l'enfant prend la parole.

Mel'čuk, I. (2001). Communivative Organization in Natural Language. Amsterdam/Philadelphia: Benjamins.

Morel, M. A., \& Danon-Boileau, L. (1998). Grammaire de l'intonation. Paris : Ophrys.

Morgenstern, A. (2006). Un JE en construction. Genèse de l'auto-désignation chez le jeune enfant. Paris : Ophrys.

Morgenstern, A., \& Parisse, Ch. (2007). «Codage et interprétation du langage spontané d'enfants de 1 à 3 ans », Corpus 6 (Interprétation, contextes, codage), 55-78.

Mosegaard Hansen, M.-B. (1998). The function of discourse particles. Amsterdam: John Benjamins.

Mousty, Ph., \& Leybaert, J. (2005). «Les Habiletés métaphonologiques : aspects théoriques et évaluation ", in Piérart, B. (éd.), Le Langage de l'enfant. Bruxelles : De Boeck, 133-143.

Oller, D. K. (2000). The Emergence of the speech capacity. Mahwah NJ: Lawrence Erlbaum.

Rebuschi, G. (2002). « Coordination et subordination : vers la co-jonction généralisée ", Bulletin de la Société de Linguistique de Paris, 97(1), $37-94$. 
Saada-Robert, M., \& Favrel, J. (2001). «Lecture/écriture émergente en situation scolaire », in M. Almgrem, A. Barrena, M. J. Ezeizabarrena, I. Idiazabal \& B. MacWhinney (eds.), Research on Child Language Acquisition: Proceedings of the 8th Conference of the International Association for the Study of Child Language. Cascadilla Press : Somerville Mass, 232-249.

Sanders, T. J. M., \& Noordman, L. G. M. (2000). « The role of coherence relations and their linguistic markers in text processing », Discourse Processes, 29(1), 37-60.

Shriberg, E., Stolcke, A., Hakkani-Tur, D., \& Tur, G. (2000). "Prosody-Based Automatic Segmentation of Speech into Sentences and Topics", Speech Communication, 32(1), 127-154.

Torterat, F. (2009). «La Dictée à l'adulte, telle que pratiquée à l'École : une approche combinée des faits grammaticaux et des phénomènes prosodiques ", Travaux linguistiques du Cerlico, 22, 293-308.

Torterat, F. (2010a). «Une analyse factorielle des productions verbales de jeunes enfants : un élément de plus en faveur des corpus longitudinaux ", Texte et Corpus, 4, 237-257.

Torterat, F. (2010b). Approches grammaticales contemporaines : constructions et opérations, Louvain-la-Neuve : Académia Bruylant (collection «Sciences du Langage : carrefours et points de vue »).

Torterat, F. (2011). " La Co-construction textuelle avec de jeunes enfants : de la phrase au texte, et vice versa ", Discours, 7 (en ligne : http://discours.revues.org/8052).

Torterat, F. (à par.). «Ce que l'Interlocution apporte à l'acquisition : l'exemple des co-constructions textuelles en classe de maternelle », in C. Douay \& D. Roulland (éds.), L'Interlocution comme paramètre, Rennes : Presses Universitaires de Rennes.

Veneziano, E., \& Hudelot, Ch. (2002). " Développement des compétences pragmatiques et théorie de l'esprit chez l'enfant : le cas de l'explication », in J. Bernicot, A. Trognon, M. Guidetti \& M. Musiol (éds.), Pragmatique et psychologie. Nancy : PUN, 215-236.

Veneziano, E. (2000). «Interaction, conversation et acquisition du langage dans les trois premières années », in M. Kail \& M. Fayol (éds.), L'Acquisition du langage (vol. 1 : Le Langage en émergence). Paris : PUF, 231-265.

Wauquier, S. (2003). «La Question du réalisme des formalisations phonologiques contemporaines : que nous apprennent les données d'acquisition ? », in J. P. Angoujard, S. Wauquier (éds.), Phonologie : champs et perspectives. Lyon : ENS éditions.

Wauquier, S. (2005). «Acquisition et développement phonologiques », in N. Nguyen, S. Wauquier \& J. Durand (éds.) Phonologie et phonétique, forme et substance. Paris : Hermès, 325-348.

Wilkins, D. P. (1992). «Interjections as deictics », Journal of Pragmatics, 18(2-3) 119-158. 